\title{
BayesianNetwork: Interactive Bayesian Network Modeling and Analysis
}

\section{DOI: $10.21105 /$ joss. 00425}

\section{Software}

- Review ct

- Repository $\boldsymbol{c}^{\boldsymbol{T}}$

- Archive [

Submitted: 15 July 2017 Published: 04 January 2018

\section{Licence}

Authors of JOSS papers retain copyright and release the work under a Creative Commons Attribution 4.0 International License (CC-BY).

\section{Paul B. Govan ${ }^{1}$}

1 GE Renewable Energy

\section{Summary}

BayesianNetwork (Govan 2017) is a shiny (Chang et al. 2017) web application for Bayesian Network modeling and analysis, providing a front-end to the bnlearn (Scutari 2009) package for Bayesian Network learning. The application includes structural learning algorithms for learning the structure of the network with support for both discrete and continuous variables, parameter learning methods for estimating the network parameters, procedures for adding evidence to the network and performing Bayesian inference, and node and network utilities for measuring the importance of connections in the network. BayesianNetwork originated as a research project for risk analysis applications, where the primary motivation was to develop an app for modeling and analyzing Bayesian Networks in an interactive environment (Govan and Damnjanovic 2016). The goal is that the package be both powerful and intuitive, serving as a tool for researchers, educators and students alike. The package documentation includes examples and additional resources for running the software.

\section{References}

Allaire, J.J., Christopher Gandrud, Kenton Russell, and CJ Yetman. 2017. NetworkD3: D3 Javascript Network Graphs from R. https://CRAN.R-project.org/package= networkD3.

Chang, Winston, and Barbara Borges Ribeiro. 2017. Shinydashboard: Create Dashboards with 'Shiny'. https://CRAN.R-project.org/package=shinydashboard.

Chang, Winston, Joe Cheng, JJ Allaire, Yihui Xie, and Jonathan McPherson. 2017. Shiny: Web Application Framework for R. https://CRAN.R-project.org/package=shiny.

Galili, Tal. 2017. Heatmaply: Interactive Cluster Heat Maps Using 'Plotly'. https: / /CRAN.R-project.org/package=heatmaply.

Ganz, Carl. 2016. "rintrojs: A Wrapper for the Intro.js Library." Journal of Open Source Software 1 (6). The Open Journal. http://dx.doi.org/10.21105/joss.00063.

Govan, Paul. 2017. BayesianNetwork: Bayesian Network Modeling and Analysis. https: / /CRAN.R-project.org/package=BayesianNetwork.

Govan, Paul, and Ivan Damnjanovic. 2016. "The Resource-Based View on Project Risk Management." ASCE Journal of Construction Engineering and Management. https: //doi.org/10.1061/(ASCE)CO.1943-7862.0001136.

Scutari, Marco. 2009. "Learning Bayesian Networks with the Bnlearn R Package." arXiv Preprint arXiv:0908.3817. 


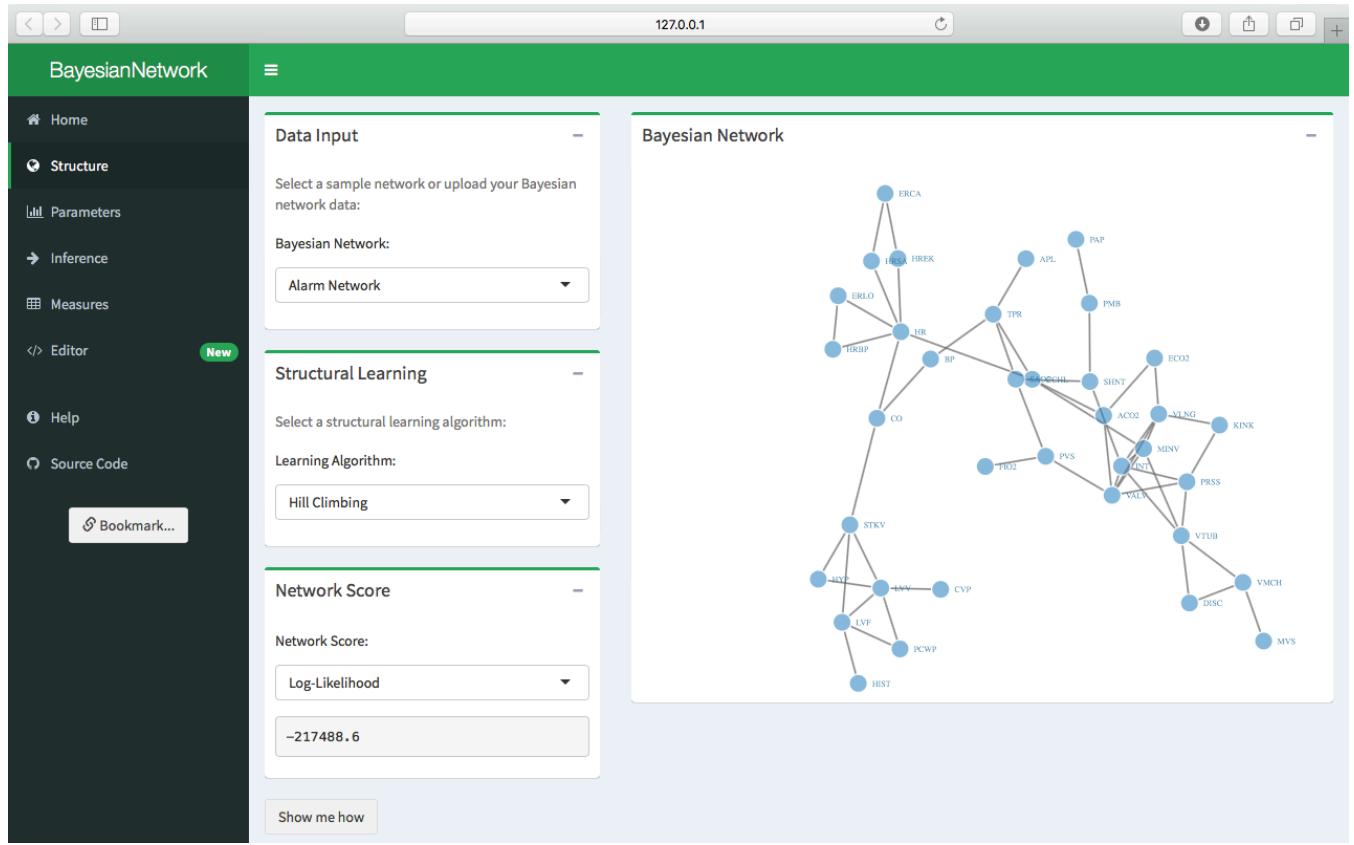

Figure 1: The Structure Tab

Trestle Technology, LLC. 2016. ShinyAce: Ace Editor Bindings for Shiny. https://CRAN. R-project.org/package=shiny Ace. 